\title{
Multiphoton Absorption Excited Upconversion Luminescence in Multimode Optical Fiber
}

\author{
M. Ferraro(1) F. Mangini( ${ }^{(2)}$, M. Zitelli( ${ }^{(1)}$, A. Niang( ${ }^{(2)}$, A. Tonello(3), V. Couderc ${ }^{(3)}$ and S. Wabnitz ${ }^{(1)}$ \\ (1) Department of Information Engineering, Electronics and Telecommunications, \\ Sapienza University of Rome, Via Eudossiana 18, 00184 Rome, Italy (mario.ferraro@uniroma1.it) \\ (2) Department of Information Engineering, University of Brescia, Via Branze 38, 25123 Brescia, Italy \\ (3) Université de Limoges, XLIM, UMR CNRS 7252,123 Avenue A.Thomas, 87060 Limoges, France
}

\begin{abstract}
Shedding light on nonlinear loss occurring close to multimode optical fiber damage threshold, we demonstrate that multiple photons are absorbed by material defects and upconverted to shorter wavelength luminescence. An array of blue emitting points results from the spatial self-imaging in graded-index fibers.
\end{abstract}

\section{Introduction}

Nonlinear pulse propagation in multimode optical fibers (MMFs) is a subject of renewed research interest, owing to their potential for optical communications and fiber lasers. Among their many advantages, MMFs may carry light beams with peak powers up to the self-focusing critical value without occurring in permanent damages. This allows to work in a regime where new nonlinear effects can be observed. Recently, we reported the presence of nonlinear losses, ascribable to multiphoton absorption (MPA) mechanisms, in graded-index (GRIN) MMFs, and analysed their effect on Raman soliton propagation ${ }^{[1]}$. Nonlinear losses introduce a fundamental, and previously undisclosed, nonlinear limitation to the energy transmission capabilities of optical fibers, and may provide an intrinsic limitation for the power scaling of spatiotemporal mode-locking with multimode fiber lasers ${ }^{[2]}$. To date, a microscopic interpretation of these intriguing phenomena is still missing. Here we fill this gap of knowledge, by linking, for the first time, MPA in the infrared spectral range with the presence of visible photoluminescence in GRIN fibers. The emission of photons with higher energy than the pump is dubbed upconversion luminescence (UL). This phenomenon finds application in a wide range of fields, ranging from physics to chemistry and biology. For the photonics community, UL represents a tool for the generation of visible laser emission with infrared optical pumping, as well as a way to extend the range of laser emission into the UV. In optical fibers, UL provides an interesting new means to extend the supercontinuum bandwidth into the UV, which is otherwise challenging to achieve by using parametric nonlinear effects.

Here, we report the experimental observation and theoretical description of visible UL in commercial GRIN MMFs, pumped by intense femtosecond infrared laser pulses. We associate the emission of visible light to the MPA of silica oxygen deficiencies related defects. The multiphoton nature of the absorption process is verified by varying the source wavelength, showing that up to 5-photon absorption mechanisms are involved. The validity of our considerations was checked by numerical simulations, finding an excellent quantitative agreement with a model based on the generalized nonlinear Schrodinger equation (GNLS).

\section{Methods}

We measured the spectrum of visible UL light side-scattered in the first few millimeters of propagation. This emitted light results from the MPA of high-peak-power femtosecond infrared pulses. Pulses of MW peak power and $180 \mathrm{fs}$ at $1030 \mathrm{~nm}$ were produced by an Yb-based laser. This can pump a hybrid optical parametric amplifier (OPA) to shift the wavelength down to $750 \mathrm{~nm}$, and reducing the pulse duration to $80 \mathrm{fs}$. The source was coupled into a commercial $50 / 125$ GRIN fiber with a relative index difference of 0.0103 . The index grading was obtained by using a parabolically decreasing concentration of Ge dopant in the core, while the cladding was made of pure silica. The $1 / \mathrm{e}^{2}$ beam diameter at the fiber input facet was equal to $7.55 \mu \mathrm{m}$ at 1030 $\mathrm{nm}$, and $11 \mu \mathrm{m}$ if compressed in time by the OPA.

\section{Experimental results}

In Fig.1a we illustrate the dependence of the measured spectrum of side-scattered light on the peak power $\left(P_{p}\right)$ of the pump laser pulses at 1030 $n$ m. A broad visible emission appears when $P_{p}$ is higher than $1.5 \mathrm{MW}$. One may distinguish the generation of three main spectral peaks at 650 , 460 , and $400 \mathrm{~nm}$, respectively. According to the literature, these peaks are attributed to oxygen deficiencies related silica defects. Specifically the red peak at $650 \mathrm{~nm}$ is due to Non-Bridging Oxygen Hole Centers (NBOHCs), while the 
a)

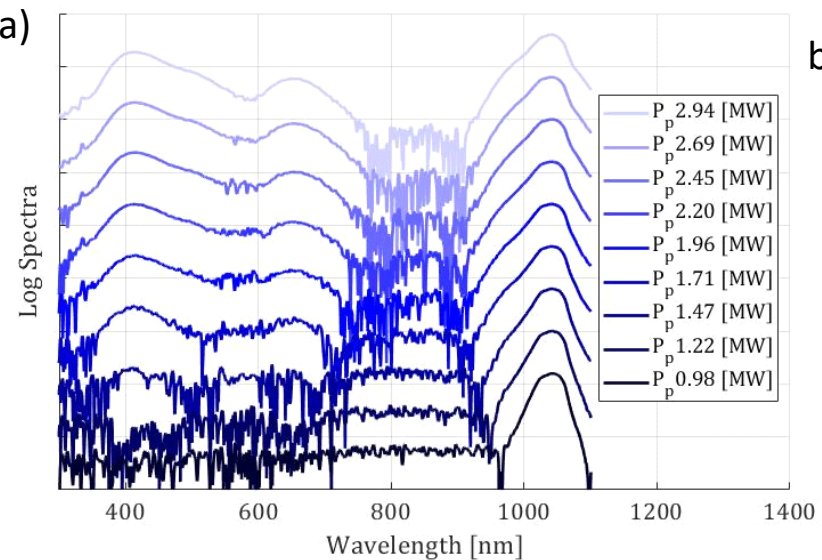

b)

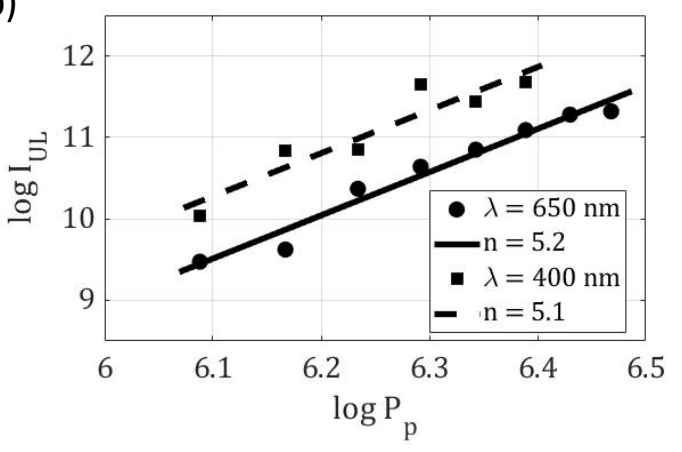

Fig. 1: (a) UL spectra in log scale when varying the input peak power. (b) Log-log plot of UL intensity as a function of the beam input peak power. Experimental data (markers) are fitted by lines, whose slope $n$ is reported in the legend.

peaks at 460 and $400 \mathrm{~nm}$ can be ascribed to Germanium - Oxygen Deficiency Centers (GeODCs $)^{[3]}$. The excitation of these defects result from MPA of the pump source, since their absorption bands are in the ultra-violet spectral range. In particular, NBOHCs and Ge-ODCs absorption peaks are so close in energy that UL generated by both defects is triggered by the same number of absorbed photons (specifically, $\mathrm{n}=5$ for a pump wave at $\lambda=1030 \mathrm{~nm}$ ). Therefore, in order to characterize the UL, as a first approximation, we may consider the presence of a single average n-photon absorption process. In this simplified model, the parameter $\mathrm{n}$ represents an effective number of absorbed photons, including all the possible contributions that are not explicitly taken into account. We may also assume that the upconversion rate is small enough, so that the UL intensity (luL) and the power are related by a power law, whose exponent is $n$ :

$$
I_{U L}=\alpha P_{p}^{n}
$$

The validity of our approximation is confirmed by the analysis illustrated in Fig.1b: we show that lut (calculated as the integral of the spectra in Fig.1a) vs. $P_{p}$ has a linear trend in the log-log plot. In particular, a slope equal to 5 is calculated for both emission bands at 400 and $650 \mathrm{~nm}$, as predicted by theory. The peak at $460 \mathrm{~nm}$ is included as the tail of that at $400 \mathrm{~nm}$.

In order to further assess the validity of the simple model (1), we varied the pump wavelength by using the OPA. In this way, the number of photons involved in the MPA process is also varied. We tuned the laser wavelength from 650 to $940 \mathrm{~nm}$. In Fig.2b we plot the Ge-ODC UL intensity as a function of the input peak power. We avoid referring to the NBOHC luminescence at $650 \mathrm{~nm}$, since it was partially covered by the spectral broadening of the source. Again, a linear dependence in the log-log plots is found. The estimation of the average number of photons that are involved in the absorption is reported in the legend. As can be seen, there is a reduction of the average number of photons with respect to the $\lambda=1030 \mathrm{~nm}$ case. Values of $\mathrm{n}$ ranging between 3.8 and 2.7 are calculated when the pump wavelength ranges between 940 and 750 $\mathrm{nm}$. These values are in agreement with predictions of our model. Indeed, we expected the number of photons to decrease down to $n=3$ for $\lambda=750 \mathrm{~nm}$, in order to match with the GeODC absorption bands. Again, the discrepancy between theoretical and experimental values is rather small, in spite of our approximation that absorption is due to a single multiphoton process. The monotonic decrease of $\mathrm{n}$ along with the laser

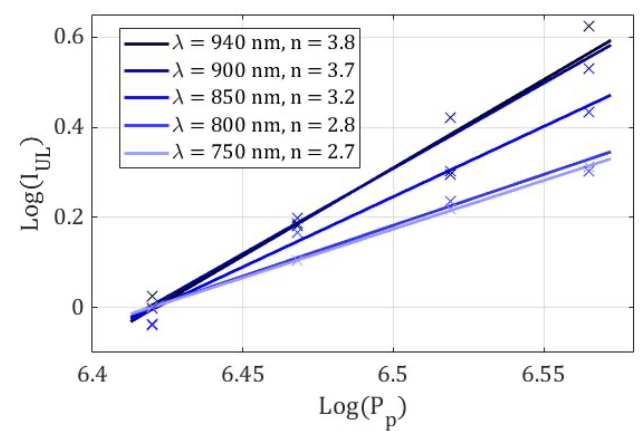

Fig. 2: Loglog plot of UL intensity vs. peak power at different pump wavelengths. Experimental data (crosses) are fitted by solid lines, whose slope $n$ is written in the legend.

wavelength is the fingerprint of the multiphoton nature of the absorption that eventually results in luminescence emission.

\section{Numerical simulation}

The estimation of the number of photons involved in the multi-photon absorption process allows us to properly model the intensity variation of UL 
along with the propagation distance. Here the key-role is played by the spatial self-imaging (SSI) effect taking place in a GRIN MMF. SSI leads to the periodic compression of the beam width along its propagation. The corresponding intensity increase enhances UL, which becomes visible to the naked eye as an array of sidescattering blue emitters. An image of the UL scattered over the first centimeter of GRIN fiber, captured by a digital microscope, is reported in Fig.3a. Equidistant emitting points are clearly recognizable. Such a geometry has already been pointed out as a result of the SSI effect in GRIN fibers $^{[4]}$. Nevertheless, the amplitude of the UL intensity variation along the propagation distance in a GRIN fiber could not be quantitatively reproduced. In Fig.3b we report the measured beam intensity variation with distance. This was obtained by a normalization of the image intensity for each pixel of propagation. The presence of a slow oscillation of the amplitude of the UL intensity peaks, whose first maximum occurs at about $3.5 \mathrm{~mm}$, is highlighted by the dashed line, which is drawn as a guide to the eye. Here the fiber length is limited to $10 \mathrm{~mm}$. By employing longer samples, we could appreciate a rapid quenching of visible light emission after the first few centimeters of fiber. To further check the relation (1) between UL and MPA, we performed a numerical simulation of spatiotemporal beam propagation in the fiber. We used a scalar 3D+1 GNLS equation including second, third, and fourth-order dispersion, Kerr, and Raman nonlinearities, as described in Ref.[1]. Random phase noise was added to the input field, in order to describe the generation of intensity speckles, and seed the generation of supercontinuum and dispersive waves. Random changes in the fiber core diameter were also considered, although their effect is negligible here because of the short fiber length. In Fig.3c we show the simulated fifth power of the light intensity peaks, along with their envelope (solid curve). As it can be seen by comparing Fig.3b and Fig.3c, there is an excellent agreement between experimental and theoretical results.

\section{Conclusion}

In conclusion, we report the observation of MPA excited UL in GRIN multimode optical fibers. We demonstrate that, depending on the source wavelength, an average number of up to $n=5$ photons simultaneously excites silica oxygendeficiencies related defects, resulting in UL. Our experimental results are in excellent agreement with numerical predictions. In perspective, UL can be exploited for extending the bandwidth of supercontinuum generation into UV frequencies.
Moreover, the appearance of UL provides a useful tool to directly monitor the presence of nonlinear losses, at peak powers close to the damage threshold of optical fibers. This paves the way for future investigations of peak power limitations in applications involving multimode fibers and lasers.

\section{Acknowledgments}

We acknowledge support from the European

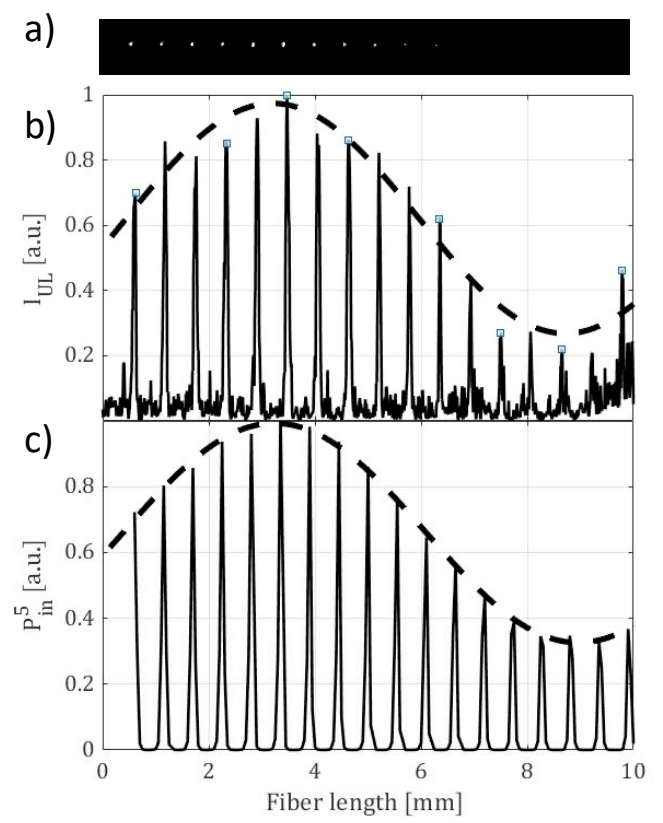

Fig. 3: (a) Microscope picture of blue light scattered from the fiber, showing periodic UL emission over the first centimeter. The figure is shown in black and white to be clearly seen once printed. (b) 1D plot of the corresponding integrated normalized scattered intensity vs. propagation distance. The dashed line is the envelope trend of the UL peaks along with the fiber. (c) 1D plot of simulated fifth power of light intensity and its envelope.

Research Council (ERC) under the European Union's Horizon 2020 research and innovation program (grant No.740355).

\section{References}

[1] M. Zitelli, F. Mangini, M. Ferraro, A. Niang, D. Kharenko, and S. Wabnitz, "High-energy soliton fission dynamics in multimode grin fiber", Opt. Express 28, 20473 (2020).

[2] L. G. Wright, D. N. Christodoulides, and F. W.Wise, "Spatiotemporal mode-locking in multimode fiber lasers", Science 358, 94 (2017).

[3] S. Girard, A. Alessi, N. Richard, L. Martin-Samos, V. De Michele, L. Giacomazzi, S. Agnello, D. D. Francesca, A. Morana, B. Winkler, I. Reghioua, P. Paillet, M. Cannas, T. Robin, A. Boukenter, and Y. Ouerdane, "Overview of radiation induced point defects in silica-based optical fibers", Reviews in Physics 4, 100032 (2019).

[4] T. Hansson, A. Tonello, T. Mansuryan, F. Mangini, M. Zitelli, M. Ferraro, A. Niang, R. Crescenzi, S. Wabnitz, and V. Couderc, "Nonlinear beam self-imaging and self-focusing dynamics in a grin multimode optical 
fiber: theory and experiments", Opt. Express 28, 24005 (2020). 